\title{
Therapeutic potential of the Nox1/4 inhibitor GKT137831 in Systemic Sclerosis
}

Heba Dosoki ${ }^{1,2}$, Muna Taha ${ }^{3}$, Hans Schnittler ${ }^{3}$, Thomas A. Luger ${ }^{1}$, Cédric Szyndralewiez, Markus Böhm

${ }^{1}$ Dept. of Dermatology, University of Münster, Germany; ${ }^{2}$ Dept. of Botany and Microbiology, Alexandria University, Alexandria, Egypt; ${ }^{3}$ Dept. of Anatomy, University of Münster, Germany;

${ }^{4}$ Genkyotex SA, Geneva, Switzerland

\section{Introduction}

The pathogenesis of systemic sclerosis (SSc) is still incompletely understood. Transforming growth factor- $\beta_{1}\left(\right.$ TGF- $\left.\beta_{1}\right)-$ mediated activation of fibroblasts and oxidative stress are crucially involved in the development of tissue fibrosis. Recently, we could show that Nox4, a member of the 7 nicotinamide adenine dinucleotide phosphate oxidase (Nox) family, is strongly upregulated by TGF- $\beta_{1}$ in normal human fibroblasts (HDFs). Genetic silencing of Nox4 as well as inhibition of Nox enzyme activity by the pan-Nox inhibitor diphenyleneiodonium neutralized this any toxicity in HDFs in vitro. Then, we examined whether GKeffect of TGF- $\beta_{1}$ (Dosoki et al. Exp. Dermatol. 2016). Here, we investigated the effect of GKT137831, a first-inclass small molecule dual-specific Nox1/4 inhibitor in HDFs in vitro as well as in vivo in experimentally induced skin fibrosis. First, we examined whether GKT137831 has T137831 can suppress TGF- $\beta_{1}$-mediated activation of HDFs in vitro. In vivo we employed the bleomycin (BLM) scleroderma mouse model to test whether GKT137831 can prevent experimentally induced skin fibrosis and is also capable to revert an already established skin fibrosis.

\section{Results}

Nox4 is up-regulated by TGF- $\beta_{1}$ in HDF
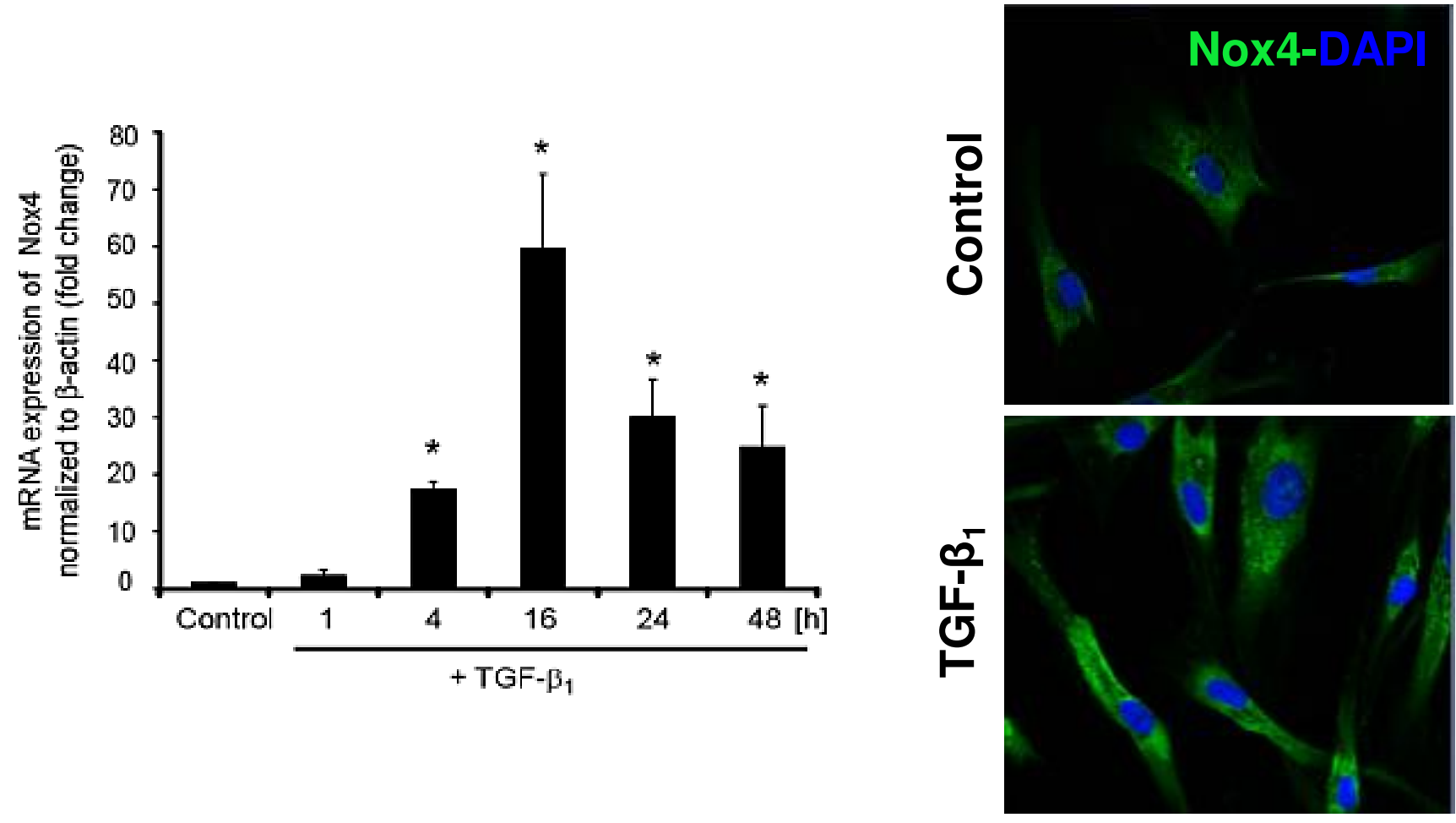

The Nox1/4 inhibitor GKT137831 does not affect cell viability and proliferation of HDFs
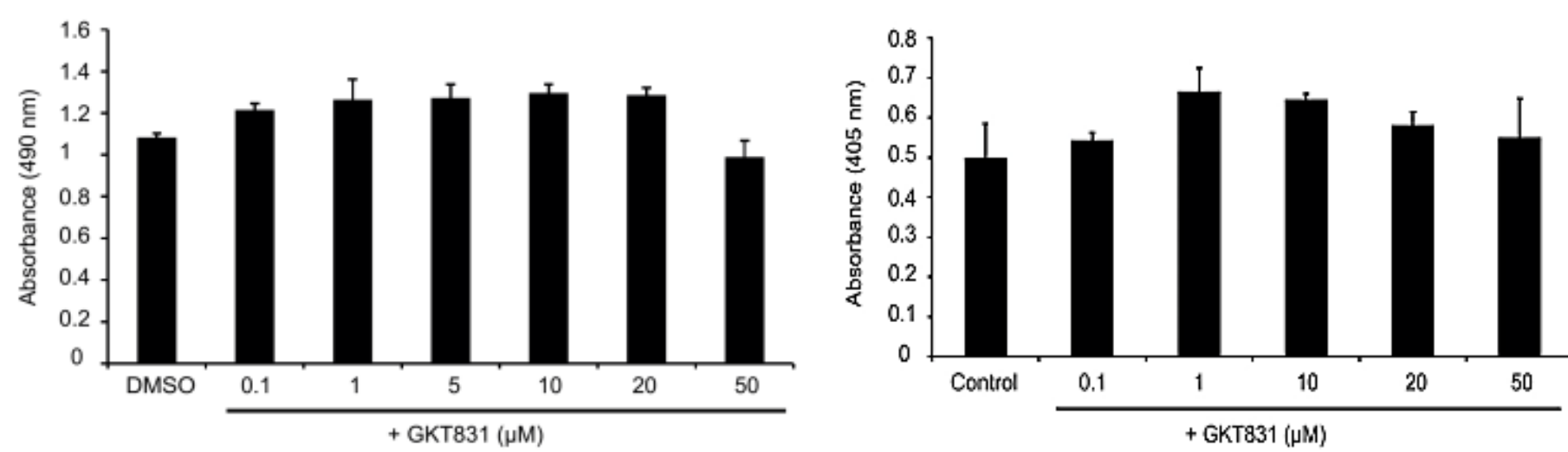

The Nox1/4 inhibitor GKT137831 reduces TGF- $\beta_{1}$-mediated upregulation of collagen type I, $\alpha$-SMA and fibronectin 1
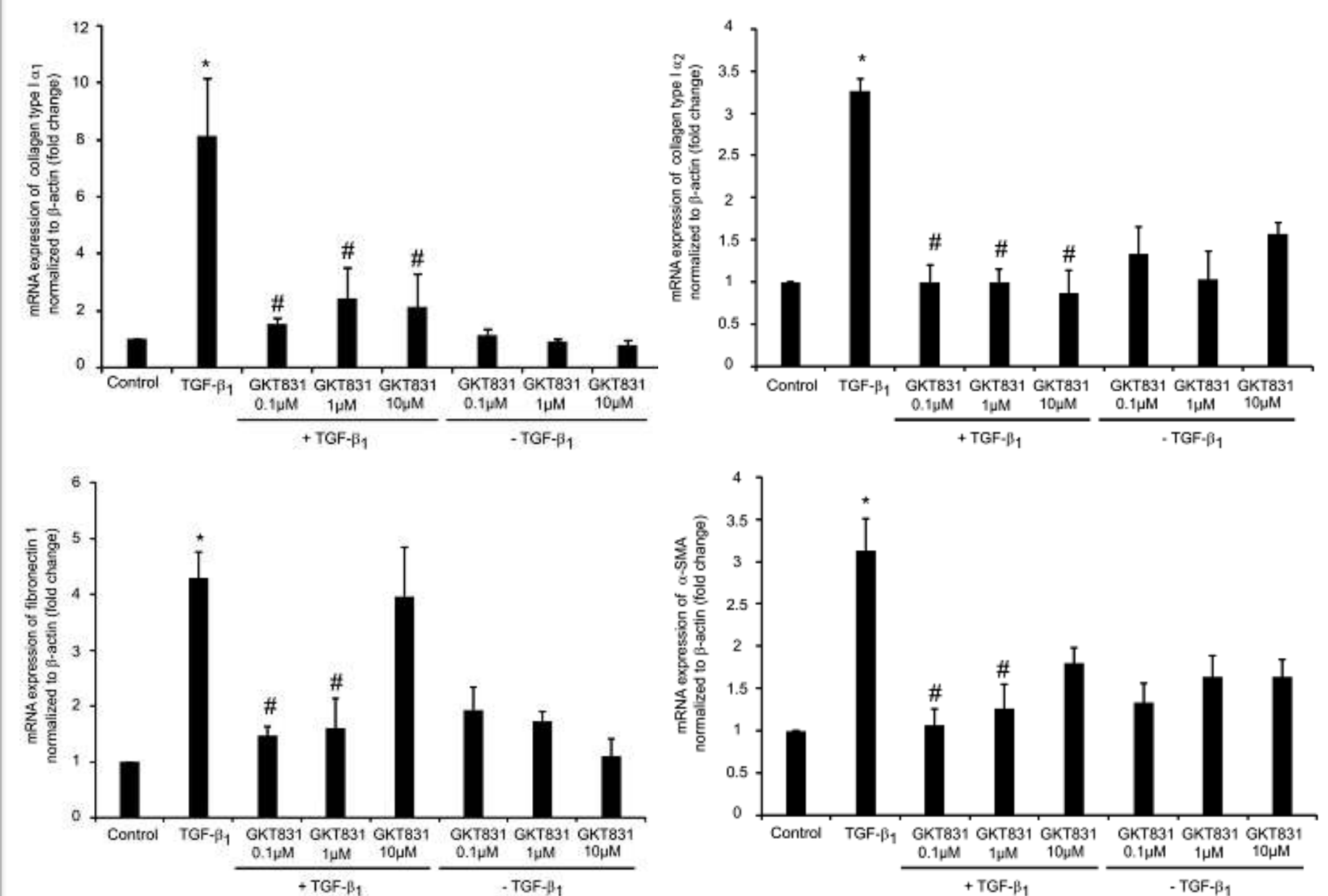
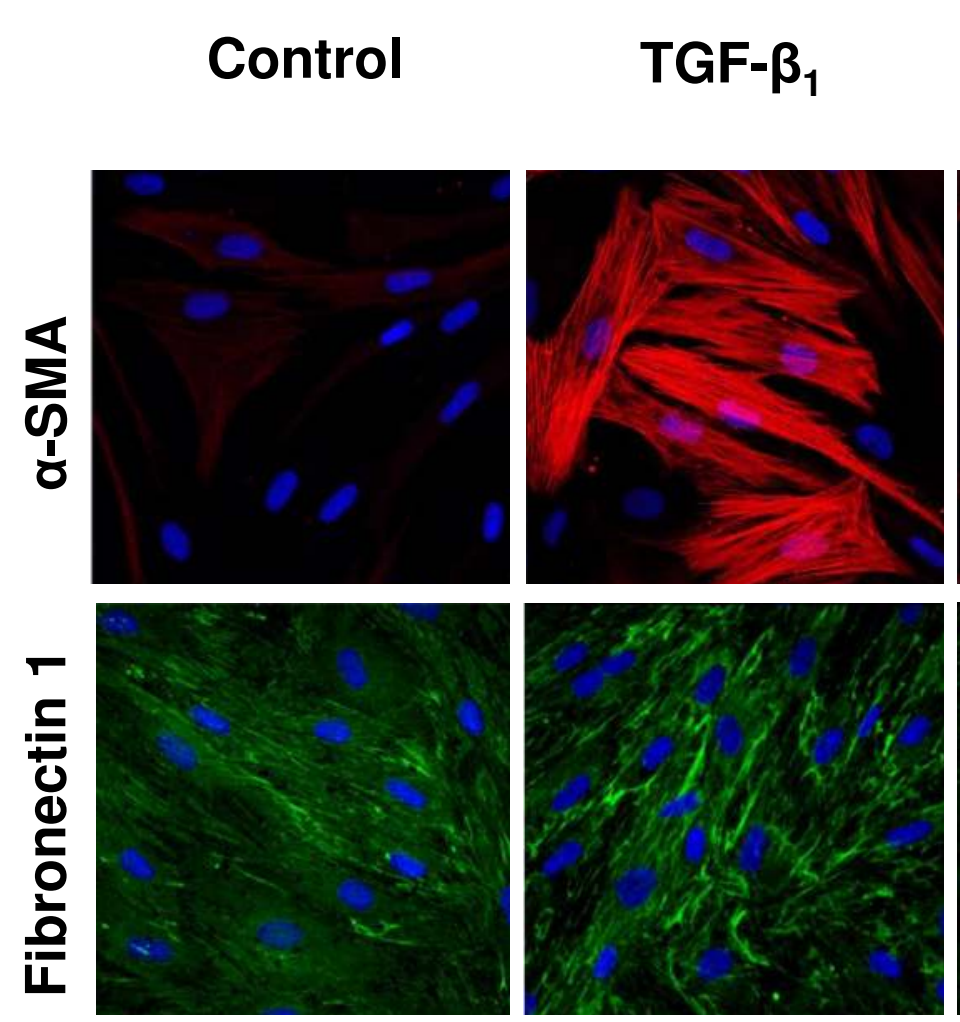

TGF- $\beta_{1}+$ GKT137831

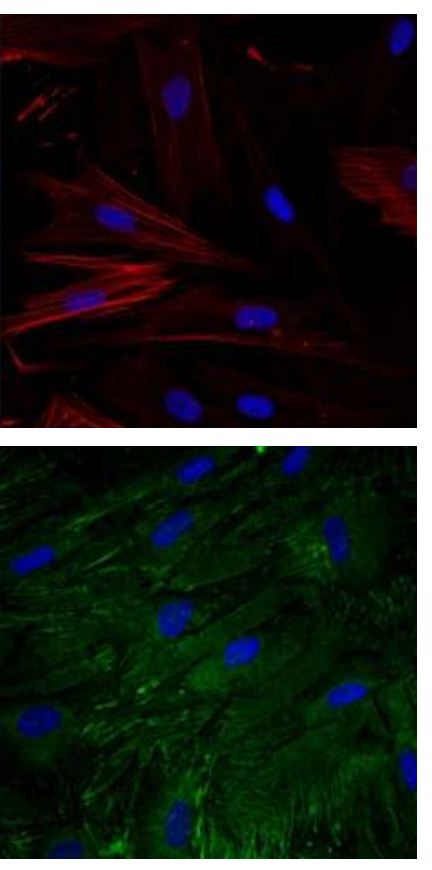

The Nox1/4 inhibitor GKT137831 attenuates bleomycininduced skin fibrosis
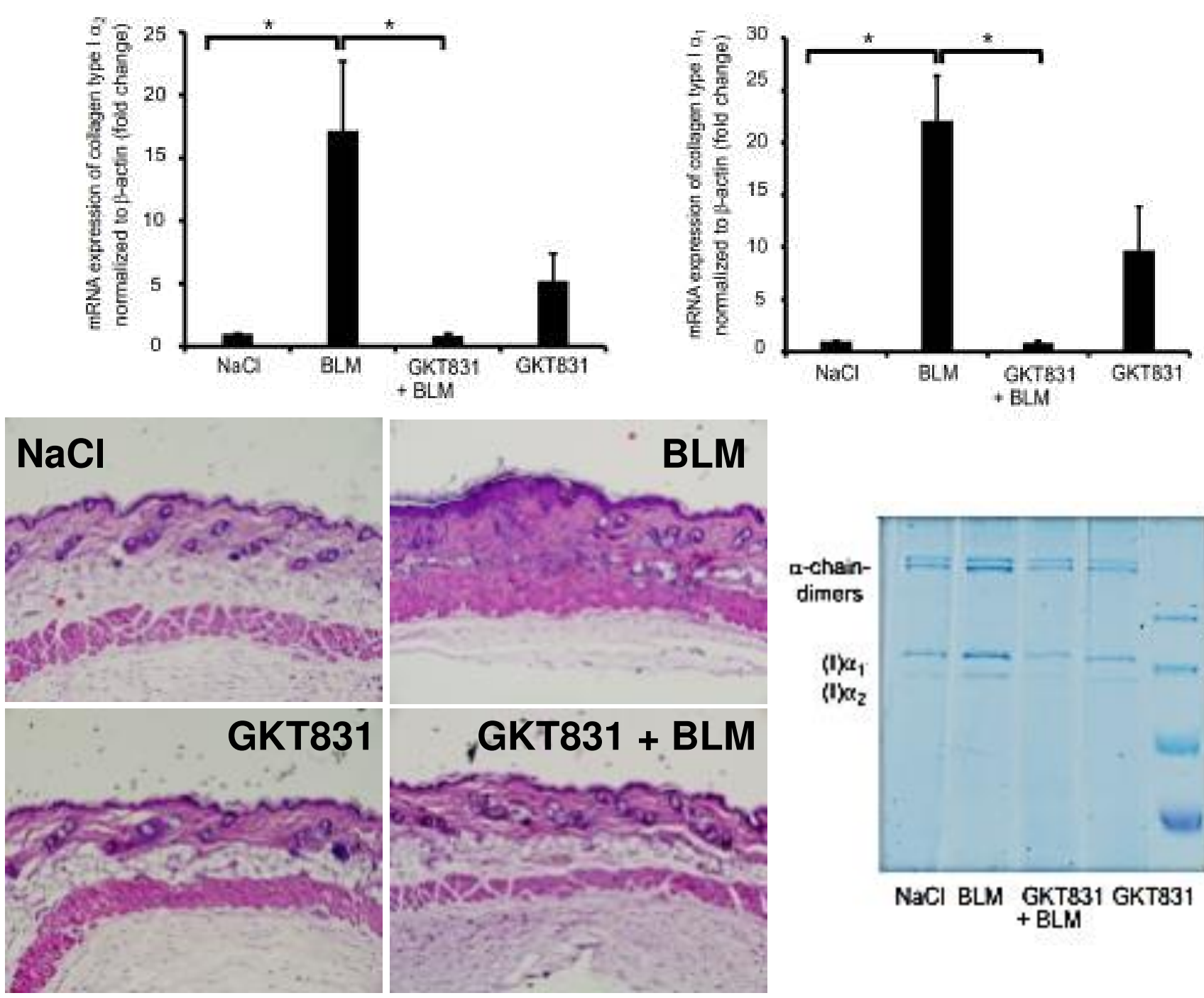

BLM
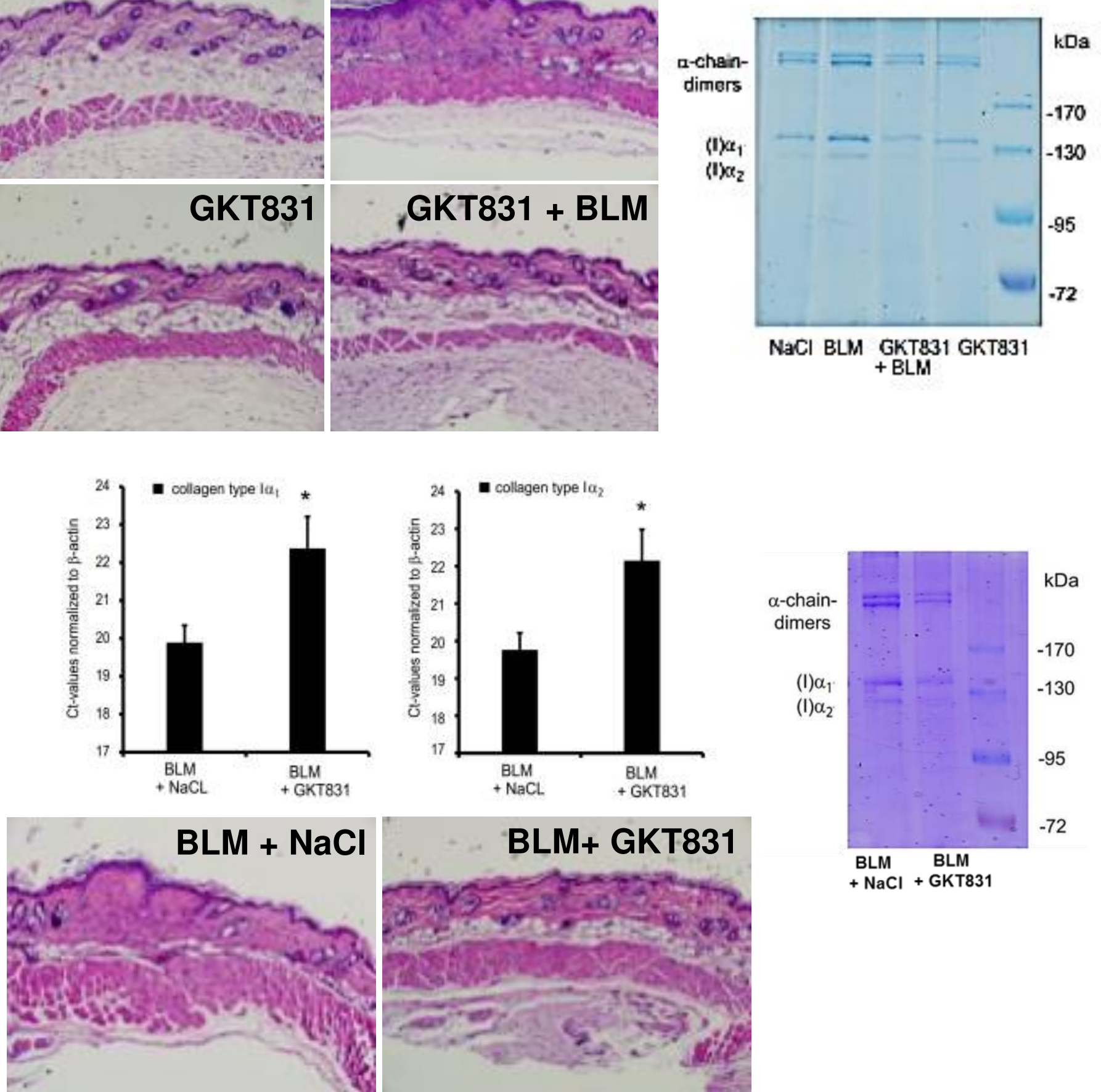

BLM+ GKT831
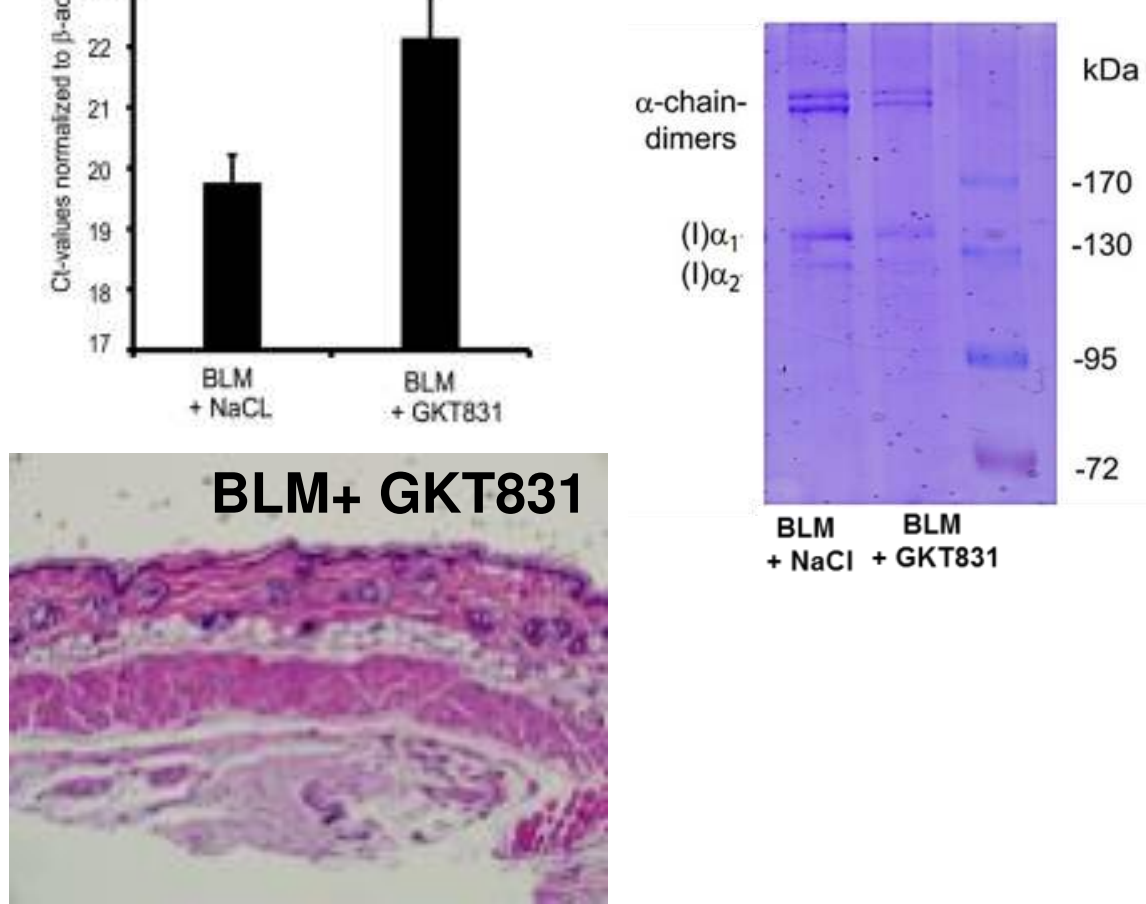

Conclusion

Our findings indicate that the Nox1/4 inhibitor GKT137831 could be a novel strategy for the treatment of fibrotic skin disease such as scleroderma

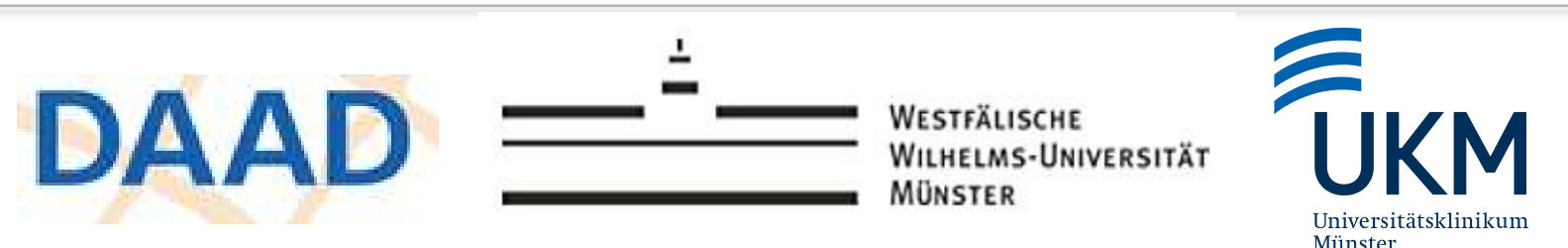

